The Open Science Foundation clandestinely abused for malicious activities

\author{
Ayumi Ikeda ${ }^{1,2^{*}}$, Fumiya Yonemitsu ${ }^{1,3^{*}}$, Naoto Yoshimura ${ }^{1,4}$, \\ Kyoshiro Sasaki ${ }^{5}$, and Yuki Yamada ${ }^{2}$ \\ ${ }^{1}$ Japan Society for the Promotion of Science \\ ${ }^{2}$ Kyushu University \\ ${ }^{3}$ Chuo University \\ ${ }^{4}$ Ritsumeikan University \\ ${ }^{5}$ Kansai University
}

*Corresponding authors

Ayumi Ikeda

Address: Graduate School of Human-Environment Studies, Kyushu University, 744 Motooka, Nishiku, Fukuoka, 819-8581, Japan.

E-mail: aikeda4444@gmail.com

TEL \& FAX: +81-92-802-5250

Fumiya Yonemitsu

Address: Faculty of Letters, Chuo University, 742-1, Higashinakano, Hachioji-shi, Tokyo, 192-0393, Japan.

E-mail: y.fumiya.0408@gmail.com

TEL \& FAX: +81-42-674-2624 


\begin{abstract}
The Open Science Framework (OSF) is an important and useful platform for researchers to practice open science. However, the OSF has recently been misused for criminal purposes, especially search boards for watching pirated copyright works and leading to phishing sites. This misuse can negatively influence the OSF server function; therefore, it is important to take measures. To protect the sound base of open science in the future, the present paper reports the two cases where the OSF has been abused for illegal activities and discusses various measures including those that the OSF management already has in place.
\end{abstract}

Keywords: open science, metascience, Open Science Framework (OSF), preregistration, open repositories 


\section{Open Science Framework}

The Open Science Framework (OSF) is a free and open-source project management platform that supports research projects (Elliott et al., 2021). The OSF is the flagship product of the Center for Open Science, Inc. (COS) whose mission is to increase the openness, integrity, and reproducibility of scientific research. This is a useful platform for researchers to share data and materials and pre-register research protocols. Indeed, the OSF has played a key role in a project on the reproducibility of psychological research (e.g., Open Science Collaboration, 2015). At the present time, researchers in various research fields, as well as psychologists, are using the OSF for managing their research projects and open collaboration. Thus, the OSF is vitally necessary for good research practices; unfortunately, here we report two cases where the OSF has been abused for illegal activities. Note that we reported this issue to the COS before making this document public, and according to the COS, they are already aware of this issue and are taking swift and concrete action to address it. The following is just a summary of what has happened up to that point we found this incident and is provided here as necessary discussion to prevent similar crimes from being committed in the future, regardless of whether the OSF is involved or not.

\section{Accessing illegal copies of copyrighted works by abusing the OSF site}

One of the authors was searching the name of the last author "Yuki Yamada" on the OSF site for purely academic purposes, and coincidently encountered a movie production starring a popular Japanese actor of the same name as him. It was created as an individual project page with embedded links to illegally uploaded sites. We were surprised when we found that page, but we suspected that this could not be the only such case. Thus, we started to look for other works that were being illegally shared through the OSF site. A number of 
such project pages were identified, and below are the details of the common method employed.

First, a person illegally uploads copyright works (e.g., commercial films) using social media as a storage site. Then, he or she sets up a project on the OSF site and makes it public with links to the illegally uploaded works in the "Description" section of the project. In other words, the OSF platform is abused as a search board for watching pirated copy, called a "leech site". For example, a search on "tumblr.com" in the OSF showed that on January 11, 2022 when we first became aware of this problem, there were 324 projects and 140 users (Fig 1). One of them is the project name “ดูหนัง $\triangleright$ Spider-Man: No Way Home【2021】 เต็มเรื่อง HD พากย์ไทย THAI" (it results in "Watch Movie $\triangleright$ Spider-Man: No Way Home [2021] Full HD Thai Dub Thai" when google translated into English). The description in this project includes a link to the illegally uploaded movie "Spider-Man: No Way Home", which is a popular movie of an American comic hero. This is just one example, but there are many other projects which have links to famous movie works and paid content.

Moreover, then, a search on "tumblr.com" in the OSF showed that as of September 27,2022 , the number of projects had decreased to 263. On the other hand, the number of users had increased to 380 . Although the change in the number of abused projects may indicate the efforts of the Center for Open Science staff, the abuse has not been eliminated. The percentage of abused projects registered in English has increased over those in languages of specific cultures. Malicious users may have changed their approach to reach more widely in this short period of time. Furthermore, we found similar cases which linked to pirated films in widely used academic research sites, such as figshare, Zenodo, Google Scholar, Academia.edu, and ORCID (search words: "1080p", "download" and "Full HD"). Researchers do not generally search for such words and thus it prevent the problem from 
coming to light easily. This information evidences that these cases are common and widespread in academic tools.

Today, there are strict punishments not only for those who upload pirated works but also for those who save them. In addition, the administrators of leech sites may also be punished when they leave the sites. In fact, there is a precedent by the European Court of Justice that providing links to illegal content constitutes copyright infringement (GS Media $v$. Sanoma Media, 2016). If the COS leaves the OSF as it is, they could be charged with a crime and the OSF could be shut down. Thus, the loss of the major platform for open science, which means the loss of a huge amount of information on study data, material, and registrations, could have a serious negative impact on the scientific community.

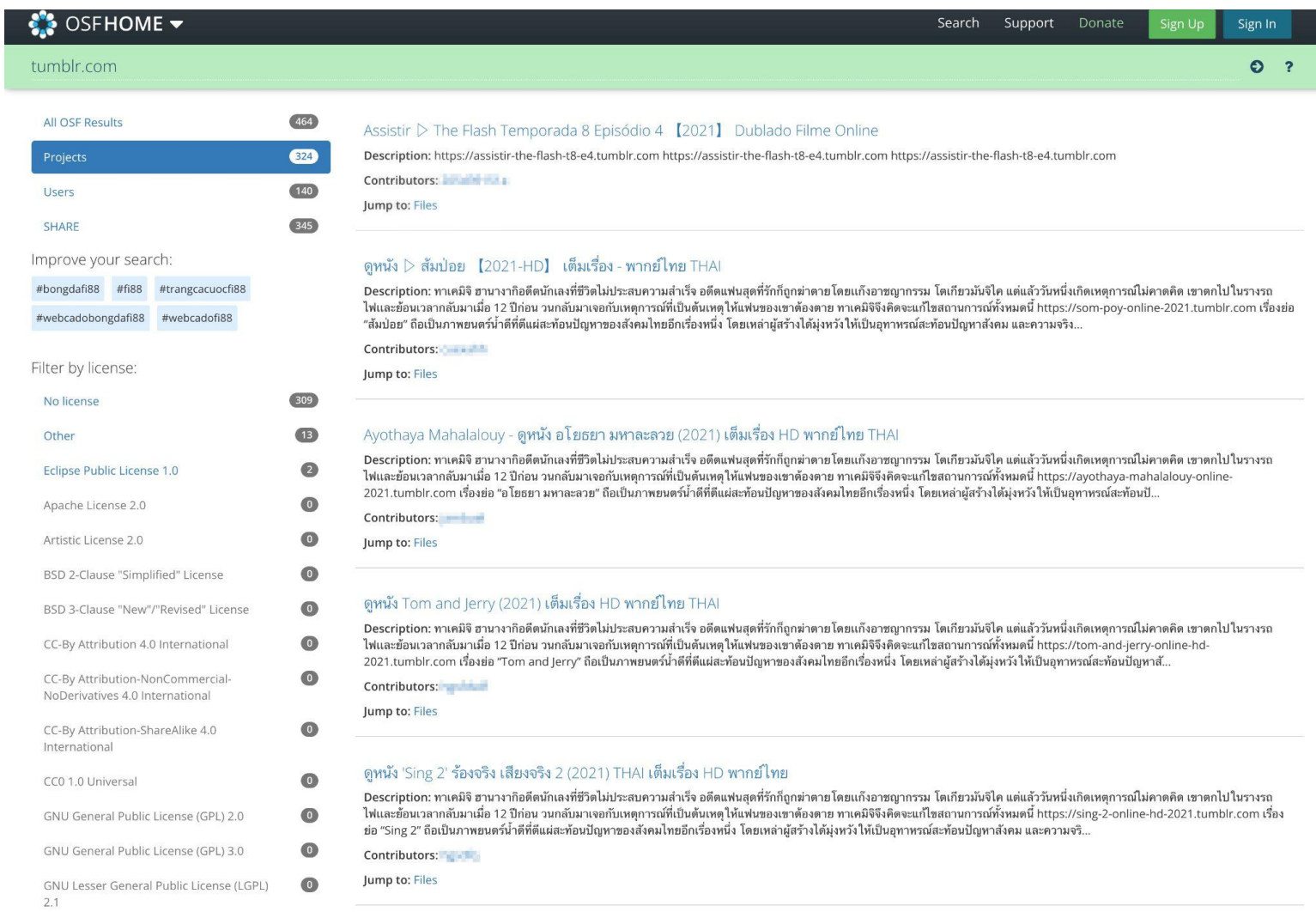

Fig 1. A search result on "tumblr.com”. Contributors' names are mosaicked by the authors. 


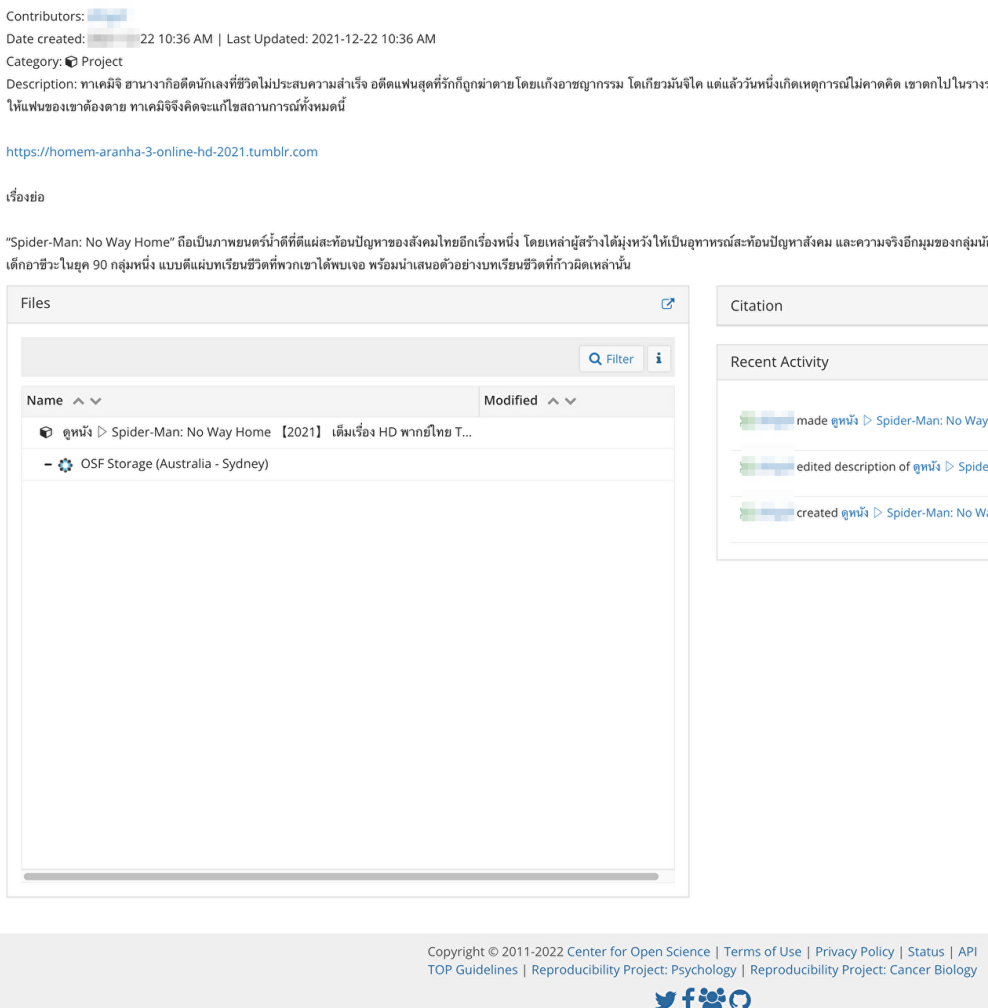

Fig 2. A project that has a link to an uploaded pirated movie. A contributor's name and the link to an uploaded pirated movie are mosaicked by the authors.

\section{Links for phishing scams also appear on the OSF}

In addition to the copyright infringement, the OSF was abused in other, more serious cybercrimes as well. We found a lot of accounts unrelated to academic research and they tried to direct users to sites where money was exchanged (Fig 3). We again conducted a search in the OSF using "tumblr.com" as a search word, which was used as a destination for illegal uploads, as introduced above. Consequently, many suspicious accounts were found. Some of the accounts had links in their personal website section that directed users to malicious websites claiming to be online casinos. These online casino sites encouraged site visitors to register as members and enter their phone numbers and credit card numbers. Such a website that withdraws important personal information from site visitors is similar to the 
characteristics of scams that are rampant on the Internet. For example, we tried searching for the site link name in the Personal website section (i.e., "happyluke withdraw money") on the Google Chrome browser. Chrome then warned us that "passwords, messages, credit cards, and other information may be stolen by malicious users." and blocked our access. This fact indicates that the OSF is abused for reaching dangerous sites which are blocked by internet security systems. Moreover, these illegal accounts and project pages are also shared on SHARE (https://share.osf.io/); the harmful effects of these abuses are spreading outside of the OSF. We just found the above cases of abuse by chance and these findings should be just the tip of the iceberg. The vulnerability of OSF to invasive uses such as illegal uploads and phishing pages indicates that the OSF site can also be used for more dangerous criminal activities, such as trading in illegal items or personal information.

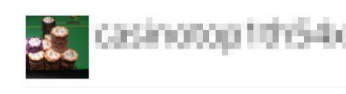

Member Since

No activity points

0 projects, 0 public
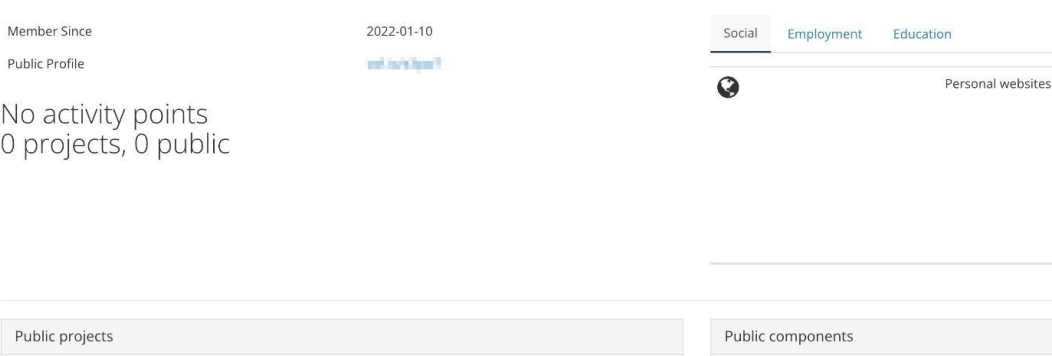

This user has no public projects.

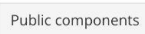

This user has no public components.

Copyright o $2011-2022$ Center for Open Science | Terms of Use | Privacy Policy | Status | AP|
TOP Guidelines | Reproducibility Project: PSychology | Reproducibility Project: Cancer Biology

- $\mathrm{f} \cos$

Fig 3. An OSF personal page of a person posting phishing sites. A contributor's name, OSF ID, and links to phishing sites are mosaicked by the authors. 
In addition to the legal issues, the fact that illegal activities are conducted on the research platform can itself be a hindrance to our research activities. For example, researchers studying films, gambling, and social media possibly use words such as a specific movie title, casino, and Tumblr in the "Description" section, and then illegal projects which contain piracy or phishing sites will become noise in search for their sane projects. Thus, accounts and projects with criminal activities prevent researchers from comfortable search activities and undermine the important functions of the $\mathrm{OSF}^{1}$. Fortunately, the oldest project with a link to the storage site, as far as we know, was built on December 28, 2021, and is still less than a month old. We should devise some countermeasures as soon as possible before serious damage occurs.

\section{Possible solutions for illegal activities at OSF}

How can we recover the soundness of the OSF site? Here, we discuss some of the mitigation strategies for this issue, which are the same as part of COS's responses to spam generally ${ }^{2}$. First of all, the COS responsible for the OSF has defined its terms of use (https://github.com/CenterForOpenScience/cos.io/blob/master/TERMS OF USE.md), and the kind of content we reported here clearly violates its Article 9 ("PERMITTED AND PROHIBITED USES"), so any account that does such activities will be banned and the content will be forcibly removed under Article 10 ("LIMITATION OF ACCESS AND REMOVAL OF CONTENT"). Obtaining help from sane users may be one way to eliminate illegal activities from the OSF site. For example, when volunteers find criminal accounts, they report them to the COS, which then bans the accounts. However, there are limits to manpower and labor in this way. Thus, it will be also necessary for the COS team to frequently patrol the OSF site using dedicated staff or automated detectors and suspend

\footnotetext{
${ }^{1}$ It says on the page of OSF HOME "Discover projects, data, materials, and collaborators on OSF that might be helpful to your own research."

2 This point has been confirmed by one of the COS's members.
} 
illegal accounts. It is possible that those who provide places where illegal activities take place could be accused (e.g., Kyodo News, 2021), and hence the COS would better take immediate and serious action.

Furthermore, restricting the way of registration might be effective. The above types of illegal activities are due mainly to the advantage of allowing anyone to create accounts. Thus, it will be effective to limit and identify those who can create the accounts by registering ID information that can identify researchers such as an email address that has a domain for academic institutions (e.g., ".ac" and “.edu") or ORCID". However, this may hinder the involvement of the public in science (i.e., citizen science) and also cause inconvenience to early-career researchers whose affiliations tend to change frequently.

Moreover, as far as piracy is concerned, attention should be paid to projects aimed for non-English speaking audiences, especially in Asia languages. A questionnaire investigation conducted by the Agency for Cultural Affairs in Japan showed that copyright damages by Asian countries are the top (Agency for Cultural Affairs, 2010). Consistent with this trend, OSF in January 2022 had a number of illegal projects using Asian languages. Such projects had decreased to some extent by October 2022 but had not been completely eradicated. For this reason, crime projects and accounts are not easily noticed by the COS, which is probably mainly composed of English-speaking staff. Hence, although the cooperation of non-English speakers is essential in this case, it is costly and difficult. Therefore, we suggest the introduction of an automatic detection system that operates in linkage with a machine translation device to automatically suspend accounts with criminal risk. Then, if the account holder launches a protest for suspension, the COS could examine the contents and determine whether to restore the account or not. This approach can be operated in languages other than English at a relatively low cost. In conclusion, not only the COS but also the entire scientific

\footnotetext{
${ }^{3}$ Note that there are also problems of empty or "ghost" ORCID accounts or the possible abuse of ORCIDs to register potentially fake elements (Teixeira da Silva, 2020a, b).
} 
community should work out good ideas and measures to prevent the OSF and other similar services and entities from being abused for criminal activities.

\section{Funding Information}

This research was supported by JSPS KAKENHI grant nos. JP19K14482 (K.S.), JP20H04581 (Y.Y.), JP20J21976 (A.I.), 21 H03784 (Y.Y., K.S.), JP21J03784 (F.Y.), JP22K13881 (K.S.), JP22K18263 (Y.Y.), JP22J01676 (N.Y.). The funders had no role in study design, data collection and analysis, decision to publish or preparation of the manuscript.

\section{Competing interests}

The authors have no competing interests to declare.

\section{Authors' contributions}

Ayumi Ikeda: Conceptualization (lead); Funding Acquisition (lead); Investigation (equal); Writing - Original Draft Preparation (equal); Writing - Review \& Editing (lead). Fumiya Yonemitsu: Conceptualization (lead); Funding Acquisition (equal); Investigation (equal); Writing - Original Draft Preparation (equal); Writing - Review \& Editing (supporting). Naoto Yoshimura: Conceptualization (supporting); Funding Acquisition (equal); Investigation (equal); Writing - Original Draft Preparation (equal); Writing Review \& Editing (supporting). Kyoshiro Sasaki: Funding Acquisition (equal); Investigation (equal); Writing - Original Draft Preparation (equal); Writing - Review \& Editing (supporting). Yuki Yamada: Funding Acquisition (equal); Investigation (equal); Supervision(lead); Writing - Original Draft Preparation (equal); Writing - Review \& Editing (supporting). 


\section{References}

Agency for Cultural Affairs (2010). [Questionnaire Survey on Piracy: Summary of Results] Kaizokuban higai nado ni kansuru anketo chousa shukei kekka gaiyo (in Japanese). $\begin{array}{llll}\text { Retrieved } \quad \text { January } & 12, & 2022, & \text { from }\end{array}$ https://www.bunka.go.jp/tokei hakusho_shuppan/tokeichosa/kaizokuban/pdf/higaicho $\underline{\text { sa houkokusho.pdf }}$

Elliott, F., Errington, T. M., Bagby, C., Frazier, M., Geiger, B. J., Liu, Y., ... \& Chen, L. (2021). OSF. Retrieved from osf.io/4znzp

Kyodo News (2021, Jun 2). Ex-illegal manga site operator slapped with three-year prison sentence. The Japan Times. Retrieved January 12, 2022, from https://www.japantimes.co.jp/news/2021/06/02/national/illegal-manga-website/

Open Science Collaboration (2015). Estimating the reproducibility of psychological science. Science, 349. doi:10.1126/ science.aac4716

Teixeira da Silva, J. A. (2020a). Failure of ORCID: 57 academics named "Beatriz". Update Dental College Journal, 10, 3-5. doi:10.3329/updcj.v10i2.50172

Teixeira da Silva, J. A. (2020b). ORCID: Issues and concerns about its use for academic purposes and research integrity. Annals of Library and Information Studies, 67, 246-250.

GS Media BV v. Sanoma Media Netherlands BV and Others, CJEU, C-160/15 (2016), https://curia.europa.eu/juris/fiche.jsf?id=C $\% 3 \mathrm{~B} 160 \% 3 \mathrm{~B} 15 \% 3 \mathrm{BRP} \% 3 \mathrm{~B} 1 \% 3 \mathrm{BP} \% 3 \mathrm{~B} 1$ $\% 3 \mathrm{BC} 2015 \% 2 \mathrm{~F} 0160 \% 2 \mathrm{FJ} \&$ oqp $=\&$ for $=\&$ mat $=$ or \&lgrec $=$ en $\& j g e=\& \mathrm{td}=\% 3 \mathrm{BALL} \& \mathrm{jur}$ $=\mathrm{C} \% 2 \mathrm{CT} \% 2 \mathrm{CF} \&$ num $=\mathrm{c}-160 \% 252 \mathrm{~F} 15 \&$ dates $=\& \mathrm{pcs}=\mathrm{Oor} \& \mathrm{lg}=\& \mathrm{pro}=\&$ nat $=$ or $\& \mathrm{cit}=\mathrm{n}$ one $\% 252 \mathrm{CC} \% 252 \mathrm{CCJ} \% 252 \mathrm{CR} \% 252 \mathrm{C} 2008 \mathrm{E} \% 252 \mathrm{C} \% 252 \mathrm{C} \% 252 \mathrm{C} \% 252 \mathrm{C} \% 252 \mathrm{C} \%$ $252 \mathrm{C} \% 252 \mathrm{C} \% 252 \mathrm{C} \% 252 \mathrm{C} \% 252 \mathrm{Ctrue} \% 252 \mathrm{Cfalse} \% 252 \mathrm{Cfalse} \&$ language $=$ en $\&$ avg $=$ $\underline{\& c i d=1694400 \# \text { section_documents }}$ (January 12, 2022) 